\title{
Treatment with the herbal medicine, naoxintong improves the protective effect of high-density lipoproteins on endothelial function in patients with type 2 diabetes
}

\author{
PU LV ${ }^{1}$, XUNLIANG TONG ${ }^{1}$, QING PENG ${ }^{1}$, YUANYUAN LIU ${ }^{1}$, HAIQIANG JIN ${ }^{1}$, \\ RAN LIU $^{1}$, WEI SUN ${ }^{1}$, BING PAN $^{2}$, LEMIN ZHENG $^{2}$ and YINING HUANG ${ }^{1}$ \\ ${ }^{1}$ Department of Neurology, Peking University First Hospital, Beijing 100034; \\ ${ }^{2}$ The Institute of Cardiovascular Sciences and Institute of Systems Biomedicine, \\ School of Basic Medical Sciences, and Key Laboratory of Molecular Cardiovascular Sciences, \\ Ministry of Education, Key Laboratory of Cardiovascular Molecular Biology and Regulatory Peptides, \\ Ministry of Health, Peking University Health Science Center, Beijing 100191, P.R. China
}

Received February 28, 2015; Accepted December 23, 2015

DOI: $10.3892 / \mathrm{mmr} .2016 .4792$

\begin{abstract}
The protective effect of high-density lipoprotein (HDL) on endothelial function is impaired in patients with type 2 diabetes mellitus (T2DM), which may result in atherosclerotic complications. Naoxintong (NXT) is a compound preparation that includes Radix Astragali, Angelicae sinensis, Radix Paeoniae Rubra and Ligusticum wallichii. It is widely administered in China to prevent atherosclerotic complications. In the present study, NXT was administered to 69 patients with T2DM. HDLs were isolated from patient blood samples prior to and following the intervention. In vitro endothelial functions of HDL, including proliferation, migration, angiogenesis, and anti-apoptosis were investigated by bromodeoxyuridine, wound healing, Transwell and Matrigel tube formation assays on human umbilical vein endothelial cells (HUVECs). The results from the present study demonstrated that HUVECs treated with HDL isolated from diabetic patients following NXT therapy exhibited increased proliferative effects (10-27\%; $\mathrm{P}<0.05)$, and improved migration ability $(15-35 \%$; $\mathrm{P}<0.05)$,
\end{abstract}

Correspondence to: Dr Yining Huang, Department of Neurology, Peking University First Hospital, 8 Xishiku Street, Beijing 100034, P.R. China

E-mail: ynhuang@sina.com

Professor Lemin Zheng, The Institute of Cardiovascular Sciences and Institute of Systems Biomedicine, School of Basic Medical Sciences, and Key Laboratory of Molecular Cardiovascular Sciences, Ministry of Education, Key Laboratory of Cardiovascular Molecular Biology and Regulatory Peptides, Ministry of Health, Peking University Health Science Center, 38 Xueyuan Street, Beijing 100191, P.R. China

E-mail: zhengl@bjmu.edu.cn

Key words: high-density lipoprotein, type 2 diabetes mellitus, traditional Chinese medicine, endothelial function anti-apoptotic function $(23-34 \% ; \mathrm{P}<0.05)$ and angiogenesis (30-54\%; $\mathrm{P}<0.001)$. Furthermore, the phosphorylation levels of Akt $(26-36 \%$; $\mathrm{P}<0.01)$ and extracellular signal-regulated kinase $(16-80 \% ; \mathrm{P}<0.01)$ were increased following NXT therapy. The present in vitro study demonstrates that the protective effect of HDL on endothelial function is markedly impaired in diabetic patients who tend to develop atherosclerosis, and the impaired function may be partly abrogated by NXT.

\section{Introduction}

Patients with type 2 diabetes have a higher risk of developing atherosclerosis due to dyslipidemia (1), which results in complications, including cardiovascular disease and stroke. High-density lipoprotein (HDL) is a critical vascular-protective factor in lipid metabolism. In addition to reverse cholesterol transportation, HDL also has other protective functions (2), including promoting nitric oxide (NO) production and flow-induced vasodilation, stimulating endothelial cell (EC) proliferation and migration, and preventing EC apoptosis. These processes are important in neovascularization and vascular repair. Furthermore, HDL stimulates capillary tube formation in vitro by increasing p42/44 mitogen-activated protein kinase (MAPK) activity (3-5). Apoptosis of ECs is triggered by exposure to inflammatory factors, resulting in disruption of the endothelial monolayer integrity. HDL inhibits tumor necrosis factor- $\alpha$ (TNF- $\alpha$ )-induced EC apoptosis (6). However, the effects of HDL may be altered or reversed, particularly in certain pathophysiological circumstances (7). Diabetic (D)-HDL exhibits reduced anti-oxidative ability and an impaired ability to stimulate NO production in ECs (7-9). In addition, a previous study demonstrated that HDL isolated from diabetic patients is dysfunctional in stimulating EC migration and proliferation due to downregulation of scavenger receptor-B1 (SR-BI) expression (10).

There is an urgent requirement to develop therapeutic agents that modulate the function of HDL particles, in addition to increasing HDL-cholesterol (HDL-c) levels. Current 
laboratory testing in clinics only provides a direct quantity of HDL-c protein, however, the antiatherosclerotic functions of HDL particles change despite no alteration in the level of HDL-c during certain disease states, including diabetes and coronary heart disease $(11,12)$. A previous study of cholesteryl ester transfer protein inhibitor, torcetrapib demonstrated a marked increase in the HDL-c level, however, increased morbidity and mortality rates were off-target effects (13). In addition to elucidating the changed functions of HDL in diabetic states, changes in HDL action in response to therapeutic strategies targeting the lipoprotein require investigation.

Herbal medicines are widely administered to treat diabetes in China. Naoxintong (NXT) is a compound of 16 herbal medicines (Radix Astragali, Radix Angelicae Sinensis, Radix Paeoniae Rubra, Radix Salviae Miltiorrhizae, Rhizoma Chuanxiong, Semen Persicae, Flos Carthami, Resina Olibani, Myrrha, Caulis Spatholobi, Radix Achyranthis Bidentatae, Ramulus Cinnamomi, Ramulus Mori, Pheretima, Scorpio and Hirudo), which has been recognized as a treatment for coronary heart disease, qi deficiency, blood stasis syndrome (in traditional Chinese medicine) and cerebrovascular diseases in clinical trials (14). It is an approved therapeutic agent for stroke by China Food and Drug Administration (15). Furthermore, NXT combined with aspirin may enhance the antiplatelet effect in patients with cardio-cerebrovascular diseases (16). In the present study, the HDL level and its functions in EC proliferation, migration, anti-apoptosis and angiogenesis during NXT intervention were investigated.

\section{Materials and methods}

Study design. Between August 1, 2014 and November 1, 2014, 30 healthy control subjects (35-70 years) and 69 patients who met the diagnostic criteria $(17,18)$ of type 2 diabetes mellitus (T2DM; 50-80 years) were recruited following informed consent. Control subjects were healthy individuals without large artery atherosclerosis, diabetics or hypertension. Carotid ultrasonography was performed on each patient. A high-resolution B-mode ultrasound machine (E8, X300PE; GE Healthcare, Little Chalfont, UK) was used to examine the common carotid arteries. The region from the beginning of the bifurcation bulb to $15 \mathrm{~mm}$ distal was examined. Atherosclerotic plaques were defined as focal structures encroaching into the arterial lumen by $0.5 \mathrm{~mm}$ or $50 \%$ of the surrounding intima-media thickness (IMT) value or an IMT value $>1.5 \mathrm{~mm}$. Carotid atherosclerosis was defined as the presence of atherosclerotic plaques in any of the aforementioned arterial segments. A total of 69 patients were divided into the diabetes with atherosclerosis group $(n=42)$ and diabetes without atherosclerosis group $(\mathrm{n}=27)$ according to the results of sonography. The present study was approved by the Institutional Review Board and the Ethics Committee of Peking University First Hospital (Beijing, China). The patients were administered NXT (Shandong Buchang Pharmaceutical Co., Ltd., Xian, China) orally at a dose of $1.2 \mathrm{~g}$ per day for 3 months. Healthy subjects without cardiovascular risk factors or disease were included in the study as baseline control and were not subjected to any intervention.

NXT (batch no. Z20025001) is composed of 16 herbs. The herbal materials were powdered and assembled into capsules. Five major compounds of NXT were analyzed by ultra-performance liquid chromatography for intestinal absorption, these were hydroxysafflor yellow A, paeoniflorin, ferulic acid, salvianolic acid B and ligustilide $(19,20)$.

Lipoprotein preparation. Following a 12-h fast peripheral venous blood samples $(4 \mathrm{ml})$ were drawn from the healthy control subjects and the patients into ethylenediamine tetraacetic acid (EDTA) tubes and kept in a refrigerator at $-80^{\circ} \mathrm{C}$ prior to the NXT intervention. In addition, samples were drawn following 90 days of treatment following a 12-h fast. Plasma $(2 \mathrm{ml})$ was isolated by centrifugation at $4^{\circ} \mathrm{C}$ and $1,000 \mathrm{x} \mathrm{g}$ for $15 \mathrm{~min}$. HDL $(1.063-1.210 \mathrm{~g} / \mathrm{ml})$ were isolated by ultracentrifugation as previously described (21). Briefly, the plasma density was adjusted to $1.3 \mathrm{~g} / \mathrm{ml}$ with $\mathrm{KBr}$ and normal saline $(1.006 \mathrm{~g} / \mathrm{ml})$ was layered over the adjusted plasma to form a discontinuous $\mathrm{NaCl} / \mathrm{KBr}$ density gradient. The tubes loaded with the sample and gradient were placed in a P40ST rotor of an ultracentrifuge (CP70MX; Hitachi, Tokyo, Japan) and were centrifuged at $350,000 \mathrm{xg}$ for $3.5 \mathrm{~h}$ at $4^{\circ} \mathrm{C}$. The HDL layer was collected. The purity of HDL was evaluated by $12 \%$ SDS-PAGE and western blot analysis using goat anti-apoA-I polyclonal antibody (DiaSorin, Stillwater, OK, USA) and quantified through the measurement of apoA-I content by nephelometry (Dimension XPand; Dade Behring, Marburg, Germany). HDL was dialyzed with $0.01 \mathrm{M}$ phosphate-buffered saline (PBS; $0.1 \%$ EDTA- $\mathrm{Na}_{2}$ ) for $72 \mathrm{~h}$. The purity of HDL and the level of apolipoprotein A-I (apoA-I) in HDL was determined.

Isolation of human umbilical vein ECs (HUVECs) and cell culture. HUVECs were isolated from umbilical veins of fresh umbilical cords as previously described (22). Umbilical cords were donated by pregnant volunteers $(n=3$; 26-28 years) following written informed consent on June 20, 2014 from the Department of Gynecology (Peking University First Hospital). The umbilical vein was washed with PBS three times and perfused with $100 \mathrm{U} / \mathrm{ml}$ collagenase type IA (Sigma-Aldrich, St. Louis, MO, USA). Following incubation at $37^{\circ} \mathrm{C}$ for $15 \mathrm{~min}$, HUVECs were collected and added to complete EC medium (ECM; Sciencell Research Laboratories, Carslbad, CA, USA). HUVECs were resuspended in ECM following centrifugation for $5 \mathrm{~min}$ at $25^{\circ} \mathrm{C}$ and $100 \mathrm{x}$ g. HUVECs were detached with $0.05 \%$ trypsin (Sciencell Research Laboratories), at a ratio of 1:3. Cells were cultured in a humidified atmosphere of $5 \% \mathrm{CO}_{2}$ at $37^{\circ} \mathrm{C}$ for 3-5 days until confluence.

Cell proliferation assay. The bromodeoxyuridine (BrdU; (Roche, Basel, Switzerland) assay was performed as described previously (15). HUVECs incubated with different types of HDL at $100 \mu \mathrm{g} / \mathrm{ml}$ apoA-I concentration for $12,24,36$ or $48 \mathrm{~h}$. The cells were labeled with BrdU labeling solution (Roche) and then fixed with paraformaldehyde (Sigma-Aldrich). Following incubation with peroxidase-conjugated anti-BrdU working solution (Roche) for $90 \mathrm{~min}$ at $37^{\circ} \mathrm{C}$, the cells were washed with washing buffer three times and substrate solution, 3,3',5,5'-tetramethylbenzidine (Roche) was added. The absorbance of each well was measured at a wavelength of $450 \mathrm{~nm}$ with an ELISA plate reader (Model 550; Bio-Rad Laboratories, Inc., Hercules, CA, USA). 
Table I. Characteristics of healthy subjects and diabetic patients.

\begin{tabular}{|c|c|c|c|}
\hline Characteristic & $\begin{array}{c}\text { Healthy } \\
\text { control }(n=30)\end{array}$ & $\begin{array}{c}\text { Diabetic } \\
\text { patients with } \\
\text { atherosclerosis }(n=42)\end{array}$ & $\begin{array}{c}\text { Diabetic } \\
\text { patients without } \\
\text { atherosclerosis }(n=27)\end{array}$ \\
\hline Age, years & $55.50 \pm 1.21$ & $66.10 \pm 1.29$ & $63.31 \pm 1.82$ \\
\hline Gender, male/female & $16 / 14$ & $17 / 13$ & $20 / 10$ \\
\hline Body mass index, $\mathrm{kg} / \mathrm{m}^{2}$ & $23.49 \pm 0.40$ & $24.34 \pm 0.31$ & $23.96 \pm 0.37$ \\
\hline Mean arterial pressure, $\mathrm{mmHg}$ & $92.92 \pm 0.80$ & $99.92 \pm 0.91$ & $101.70 \pm 1.44$ \\
\hline Fasting glucose, mmol/1 & $4.65 \pm 0.15$ & $7.87 \pm 0.40^{\mathrm{a}}$ & $8.70 \pm 0.61^{\mathrm{a}}$ \\
\hline Hemoglobin A1c, $\%$ & $4.50 \pm 0.50$ & $9.49 \pm 0.20^{\mathrm{a}}$ & $9.05 \pm 0.26^{\mathrm{a}}$ \\
\hline LDL cholesterol, mmol/1 & $2.30 \pm 0.30$ & $2.76 \pm 0.15$ & $2.74 \pm 0.14$ \\
\hline HDL cholesterol, mmol/l & $1.45 \pm 0.10$ & $1.24 \pm 0.06^{\mathrm{a}}$ & $1.22 \pm 0.07^{\mathrm{a}}$ \\
\hline Triglycerides, mmol/1 & $1.67 \pm 0.35$ & $1.98 \pm 0.29$ & $1.88 \pm 0.58$ \\
\hline Total cholesterol, mmol/1 & $3.80 \pm 0.19$ & $4.45 \pm 0.19$ & $4.54 \pm 0.18$ \\
\hline Anti-hypertensive agents & $0 / 30$ & $15 / 30$ & $14 / 30$ \\
\hline Statin therapy & $0 / 30$ & $5 / 30$ & $4 / 30$ \\
\hline Anti-diabetes therapy & $0 / 30$ & $30 / 30$ & $30 / 30$ \\
\hline
\end{tabular}

Values are expressed as mean \pm standard deviation or number of patients. ${ }^{\mathrm{a}} \mathrm{P}<0.01$ vs. the healthy control (Student's t-test). LDL, low-density lipoprotein; HDL, high-density lipoprotein.

Wound healing assay. Wound healing and Transwell assays were used to analyze HUVEC migration. The procedure was conducted as described previously (10). The HUVEC monolayer was scraped using a pipette tip and then incubated with EC medium containing $1 \%$ bovine serum albumin (BSA) alone or with different types of HDL at an apoA-I concentration of $100 \mu \mathrm{g} / \mathrm{ml}$ for $18 \mathrm{~h}$. Images of the migrated cells were captured and quantified in 10 random fields (CK40; Olympus Corp., Tokyo, Japan), and results were repeated for 15 individuals from each group.

Transwell experiments. Quantitative migration assays of HUVECs were performed using a modified Boyden chamber (Minicell; EMD Millipore, Billerica, MA, USA) with an 8.0- $\mu \mathrm{m}$ pore polycarbonate filter inserted. Cells were treated as described in the wound healing assay. Following migration for $5 \mathrm{~h}$, migrated cells were fixed and stained with crystal violet. Migrated cells were visualized in 10 high-power fields (magnification, x100), and images were captured for each chamber and quantified in 5 random fields. Results were repeated for 10 individuals from each group.

Anti-TNF- $\alpha$-induced apoptosis assay. HUVECs were seeded at a density of $5 \times 10^{5}$ cells/well in 6-well plates and cultured to $70 \%$ confluence. HUVECs were pretreated with serum-free ECM with different types of HDL at $100 \mu \mathrm{g} / \mathrm{ml}$ apoA-I concentration for $18 \mathrm{~h}$, and then incubated in the presence of TNF- $\alpha$ (200 U/ml; BD Biosciences, Franklin Lakes, NJ, USA) for $24 \mathrm{~h}$. Cells were washed with PBS three times and fixed with $4 \%$ paraformaldehyde for $10 \mathrm{~min}$ at room temperature. Subsequently, cells were washed twice with PBS and stained with Hoechst 33258 (Beijing TransGen Biotech Co., Ltd., Beijing, China) for $20 \mathrm{~min}$ at room temperature. Following three washes with PBS, cells were observed using a microscope (LSM 510; Zeiss GmbH, Jena, Germany).
Caspase-3 activity assay. Caspase-3 activity in the cells was detected using a Caspase-3 Colorimetric assay kit (Beyotime Institute of Biotechnology, Haimen, China). Cells were treated as described above. Cell lysates were centrifuged at $10,000 \mathrm{xg}$ for $15 \mathrm{~min}$ at $4^{\circ} \mathrm{C}$, the supernatants were collected and the protein concentration determined by BCA Protein assay kit (Pierce Biotechnology, Inc., Rockford, IL, USA). Cellular extracts $(30 \mu \mathrm{g})$ were then incubated in a 96-well microtitre plate with $20 \mathrm{ng}$ Ac-DEVD-pNA (Beyotime Institute of Biotechnology) for $2 \mathrm{~h}$ at $37^{\circ} \mathrm{C}$. Caspase activity was measured by cleavage of the Ac-LEVD-pNA substrate to pNA, the absorbance of which was measured at a wavelength of $405 \mathrm{~nm}$. Relative caspase activity was calculated as a ratio of emission of fluorescence of treated cells to untreated cells.

Tube formation assays. The effects of different HDL treatments on in vitro angiogenesis were assessed by Matrigel tube formation assay as described previously (23). Each well of pre-chilled 96-well plates was coated with $50 \mu 1$ Matrigel (BD Biosciences) and incubated at $37^{\circ} \mathrm{C}$ for $30 \mathrm{~min}$ for solidification. Following removal of any fluid, $\sim 4 \times 10^{4}$ HUVEC cells were seeded and cultured in free ECM containing different types of HDL at a concentration of $100 \mu \mathrm{g} / \mathrm{ml}$ apo-AI. Images were digitally captured using an Olympus microscope (Olympus Corp.) $4 \mathrm{~h}$ after seeding. Tube formation was assessed by counting the length of tubes from 10 random fields (magnification $\mathrm{x} 100)$. Results were repeated for 10 individuals from each group.

Western blotting analysis. HUVECs were cultured in 12-well plates and treated with ECM or the various types of HDL at an apoA-I concentration of $100 \mu \mathrm{g} / \mathrm{ml}$ for $15 \mathrm{~min}$ for the indicated times. Following administration of HDL for $15 \mathrm{~min}$, the cells were lysed with radioimmunoprecipation assay buffer [(Beijing TransGen Biotech Co., Ltd.) 50 mM Tris, pH 7.4, 150 mM 


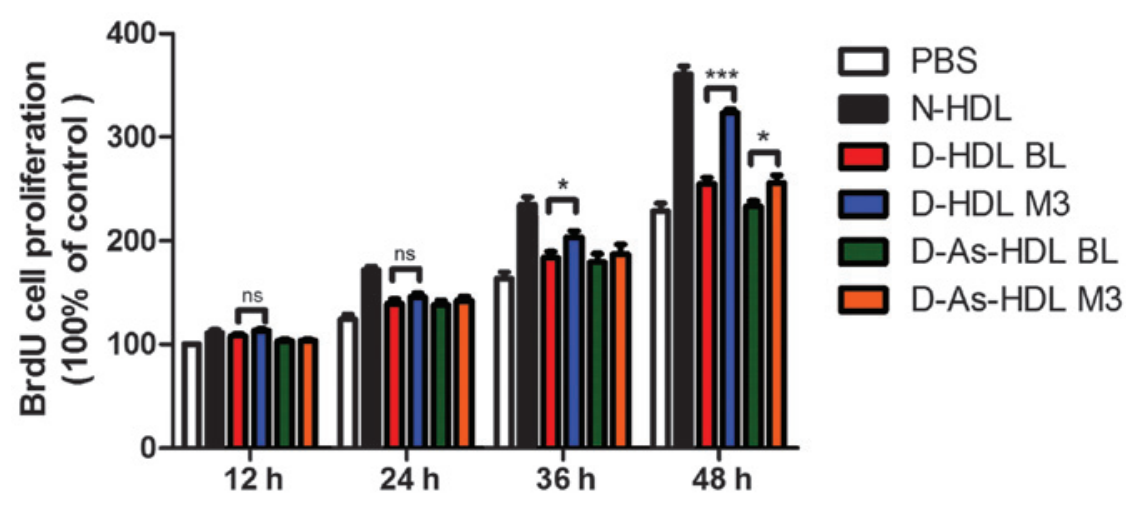

Figure 1. NXT therapy improves proliferative effects of HDL on endothelial cells in diabetic patients. Human umbilical vein endothelial cells were treated with N-HDL, D-HDL BL, D-HDL M3, D-As-HDL BL, D-As-HDL M3 (n=10 each) in $100 \mathrm{mg} / \mathrm{ml}$ apolipoprotein A-I for 12, 24, 36 or $48 \mathrm{~h}$ and cell proliferation was analyzed using a BrdU proliferation assay. Data are presented as mean \pm standard error of the mean; ${ }^{*} \mathrm{P}<0.05,{ }^{* * *} \mathrm{P}<0.001$ (Student's $\mathrm{t}$-test). NXT, naoxintong; HDL, high-density lipoprotein; N, normal; D, diabetic; BL, baseline; M3, 3 months of NXT; BrdU, bromodeoxyuridine; PBS, phosphate-buffered saline; As, atherosclerosis.

A

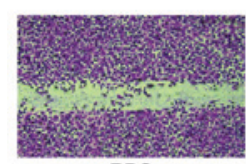

PBS

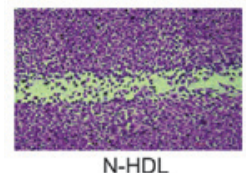

N-HDL

C
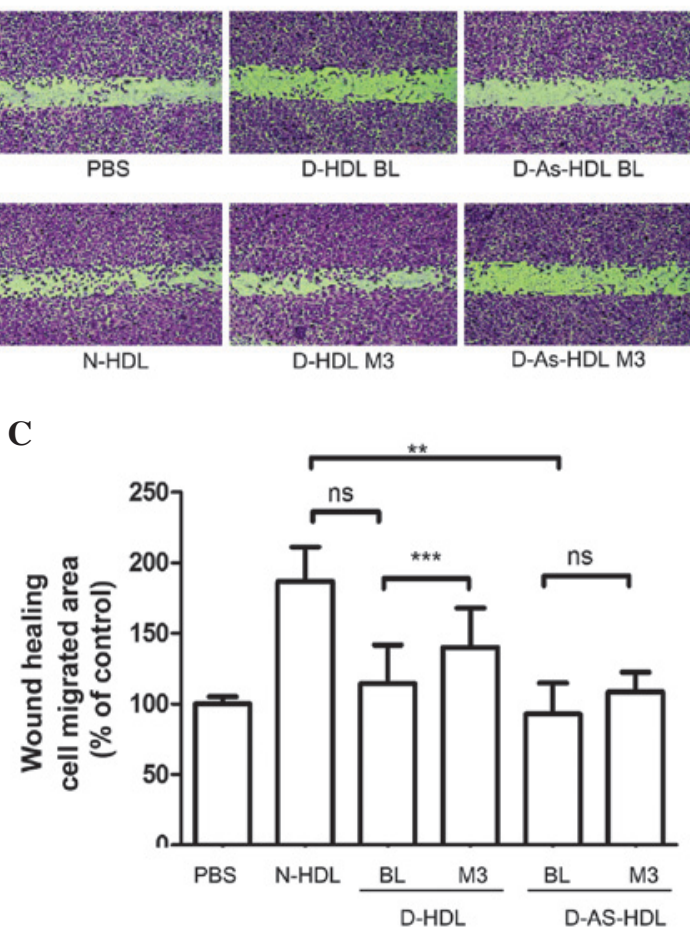

D-HDL BL


B

D

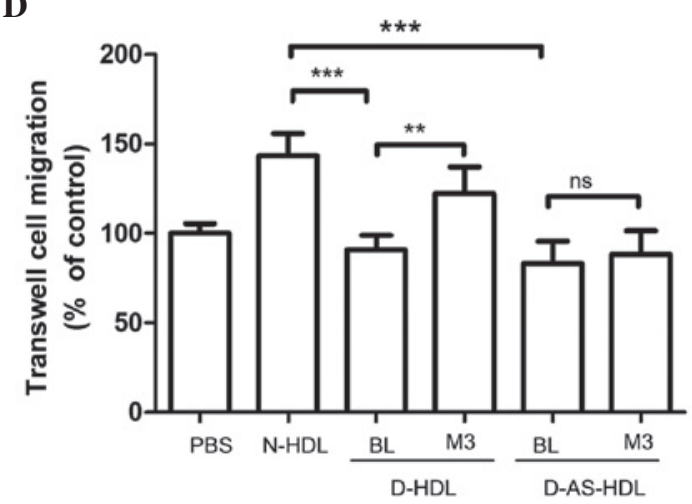

Figure 2. NXT therapy improves the effects of HDL on endothelial migration in diabetic patients. HUVECs were treated with N-HDL, D-HDL BL, D-HDL M3, D-As-HDL BL, D-As-HDL M3 in $100 \mathrm{mg} / \mathrm{ml}$ apolipoprotein A-I. (A) Representative images of HUVEC migration under different HDL treatments in the wound healing assay. (B) Representative images of HUVEC migration under different HDL treatments in wound healing assay. (C) Quantification of wound healing assay ( $\mathrm{n}=15$ each). (D) Cell migration quantification based upon an 8 -h incubation in the Transwell migration assay $\left(\mathrm{n}=10\right.$ each). ${ }^{* *} \mathrm{P}<0.01,{ }^{* * * *} \mathrm{P}<0.001$ (Student's t-test). NXT, naoxintong; HDL, high-density lipoprotein; N, normal; D, diabetic; BL, baseline; M3, 3 months of NXT; PBS, phosphate-buffered saline; As, atherosclerosis; HUVEC, human umbilical vein endothelial cell; ns, no significance.

$\mathrm{NaCl}, 1 \% \mathrm{NP}-40,0.5 \%$ sodium deoxycholate, $0.1 \%$ sodium dodecyl sulfate (SDS), $0.1 \%$ EDTA and $1 \%$ Triton X-100]. Cell protein samples were separated by centrifugation $\left(4^{\circ} \mathrm{C}\right.$, $10,000 \mathrm{x} \mathrm{g}$ for $20 \mathrm{~min}$ ), and the concentration was detected by Coomassie brilliant blue (Sigma-Aldrich). The protein samples $(40 \mu \mathrm{g})$ were subjected to $10 \%$ SDS-polyacrylamide gel (Beijing TransGen Biotech Co., Ltd.) electrophoresis (100 V for $120 \mathrm{~min}$ ) and transferred onto nitrocellulose membranes (EMD Millipore) according to standard protocols. The membranes were blocked for $2 \mathrm{~h}$ with $1 \%$ BSA containing $0.05 \%$ Tween 20 in Tris-buffered saline (Beijing TransGen Biotech Co., Ltd.). Membranes were incubated with primary antibodies, including monoclonal rabbit anti-human phosphorylated (p)-Akt (phospho S47; cat. no. ab81283; Abcam, Cambridge, MA, USA; 1:1,000), monoclonal rabbit p-extracellular signal-regulated kinase (ERK)1/2 (Cell Signaling Technology, Inc.; 1:1,000; T202/Y204; cat. no. 4377), monoclonal rabbit anti-ERK1/2 (cat. no. 9102; Cell Signaling Technology, Inc.; 1:1,000) and rabbit monoclonal anti-AKT1/2/3 antibody (cat. no. ab179463; Abcam) overnight at $4^{\circ} \mathrm{C}$ followed by horseradish peroxidase-conjugated 
Table II. Characteristics of diabetic patients prior to and following naoxintong treatment.

\begin{tabular}{|c|c|c|c|c|c|c|}
\hline \multirow[b]{2}{*}{ Characteristic } & \multicolumn{3}{|c|}{$\begin{array}{l}\text { Diabetic patients with } \\
\text { atherosclerosis }(n=42)\end{array}$} & \multicolumn{3}{|c|}{$\begin{array}{l}\text { Diabetic patients without } \\
\text { atherosclerosis }(n=27)\end{array}$} \\
\hline & Prior to & Following & P-value & Prior to & Following & P-value \\
\hline Fasting glucose, mmol/l & $7.87 \pm 0.40$ & $7.06 \pm 0.23$ & 0.09 & $8.70 \pm 0.61$ & $7.80 \pm 0.51$ & 0.27 \\
\hline Hemoglobin A1c, \% & $9.49 \pm 0.21$ & $7.06 \pm 0.23$ & $<0.01$ & $9.05 \pm 0.26$ & $7.29 \pm 0.26$ & $<0.01$ \\
\hline LDL-c, mmol/1 & $2.76 \pm 0.15$ & $2.38 \pm 0.10$ & 0.04 & $2.74 \pm 0.14$ & $2.53 \pm 0.34$ & 0.52 \\
\hline HDL-c, mmol/1 & $1.24 \pm 0.06$ & $1.29 \pm 0.05$ & 0.19 & $1.22 \pm 0.07$ & $1.21 \pm 0.07$ & 0.95 \\
\hline Triglycerides, mmol/1 & $1.98 \pm 0.29$ & $2.02 \pm 0.39$ & 0.94 & $1.88 \pm 0.58$ & $1.63 \pm 0.27$ & 0.81 \\
\hline Total cholesterol, mmol/1 & $4.45 \pm 0.19$ & $4.58 \pm 0.19$ & 0.65 & $4.54 \pm 0.18$ & $4.05 \pm 0.45$ & 0.24 \\
\hline
\end{tabular}

Values are expressed as means \pm standard deviation or number of patients. LDL, low-density lipoprotein; HDL, high-density lipoprotein.

A

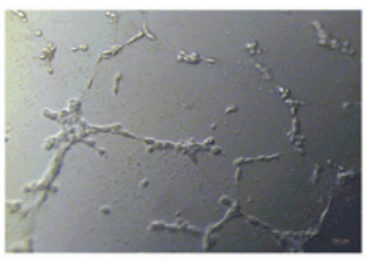

PBS



$\mathrm{N}-\mathrm{HDL}$

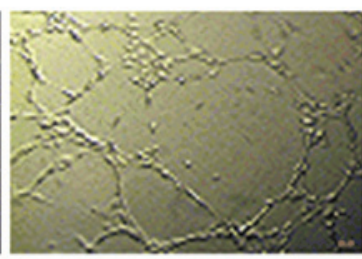

D-HDL BL

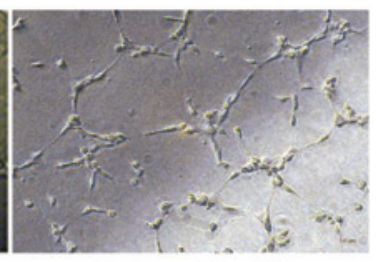

D-As-HDL BL

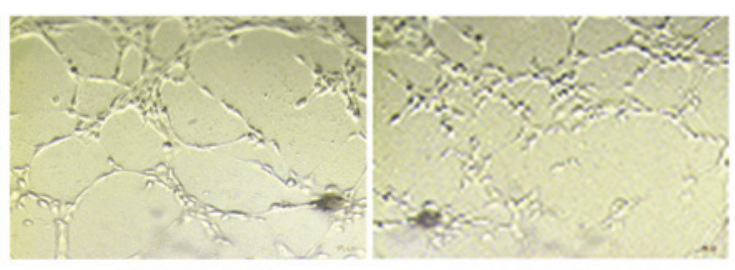

D-HDL M3

D-As-HDL M3

$\mathbf{B}$

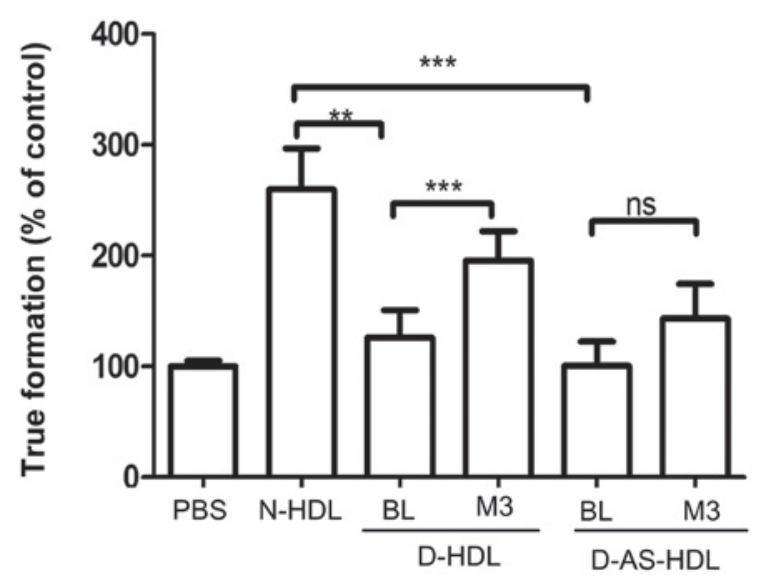

Figure 3. NXT therapy improves the endothelial-associated angiogenesis effect of HDL in diabetic patients. (A) N-HDL, D-HDL BL, D-HDL M3, D-As-HDL BL, D-As-HDL M3 was added to each well in $100 \mathrm{mg} / \mathrm{ml}$ apolipoprotein A-I and images of tube formation were captured after 3 h. (B) The tube formation was evaluated by counting 10 random fields (magnification, x100). ${ }^{* *} \mathrm{P}<0.01,{ }^{* * *} \mathrm{P}<0.001$ (Student's t-test). NXT, naoxintong; HDL, high-density lipoprotein; N, normal; D, diabetic; BL, baseline; M3, 3 months of NXT; PBS, phosphate-buffered saline; As, atherosclerosis; ns, no significance.

goat-anti-rabbit IgG secondary antibody (cat. no. SC-2004; Santa Cruz Biotechnology, Inc., Dallas, TX, USA; 1:1,000). Protein bands were detected by electrochemiluminescence using the Super Signal West Pico kit (Pierce Biotechnology, Inc.).
Statistical analysis. All experiments were reproduced in triplicate. In the majority of experiments, the control sample was defined as $100 \%$, and for each sample, increase or decrease of the percentage in other samples compared with the control 
A



PBS

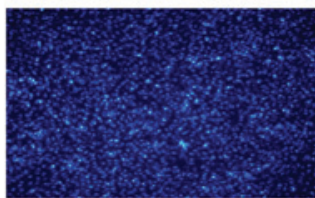

$\mathrm{N}-\mathrm{HDL}$

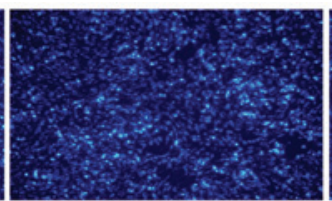

D-HDL BL



D-HDL M3

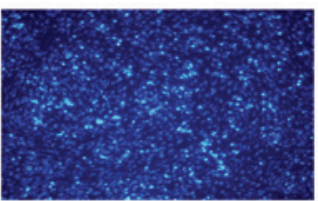

D-As-HDL BL

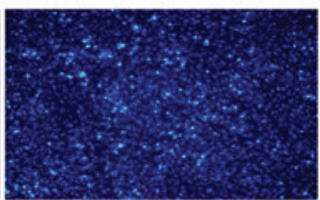

D-As-HDL M3

B

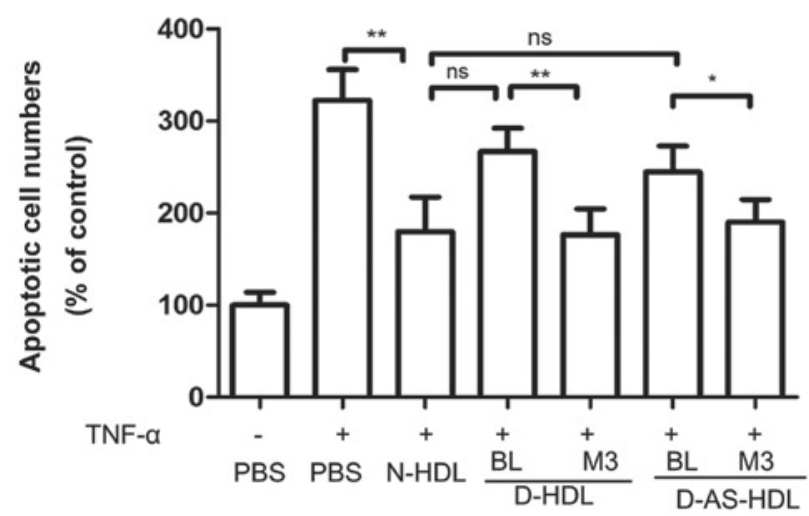

C

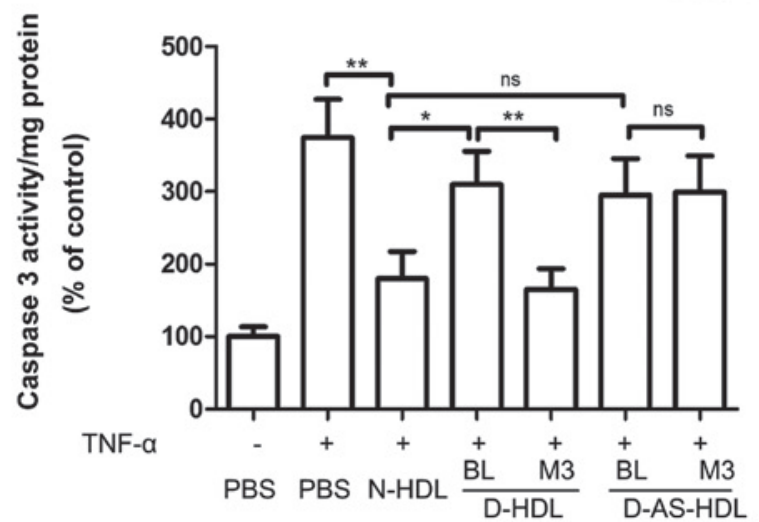

Figure 4. NXT therapy improves the anti-apoptotic effects of D-HDL for HUVECs following TNF- $\alpha$ treatment. HUVECs were pre-incubated with N-HDL, D-HDL BL, D-HDL M3, D-As-HDL BL, D-As-HDL M3 in $100 \mathrm{mg} / \mathrm{ml}$ apolipoprotein A-I for $18 \mathrm{~h}$. TNF- $\alpha$ was added to each well and incubated for $24 \mathrm{~h}$. (A) Representative images of apoptotic cells stained by Hoechst 33258 (objective lens magnification, x100). (B) The apoptotic cells were evaluated by counting 10 random fields. (C) Caspase-3 activity in cell lysates was determined. ( $\mathrm{n}=6) .{ }^{*} \mathrm{P}<0.05,{ }^{* *} \mathrm{P}<0.01$ (Student's t-test). NXT, naoxintong; HDL, high-density lipoprotein; HUVECs, human umbilical vein endothelial cells; TNF- $\alpha$, tumor necrosis factor- $\alpha$; N, normal; D, diabetic; BL, baseline; M3, 3 months of NXT; PBS, phosphate-buffered saline; As, atherosclerosis; ns, no significance.

was calculated. Data were presented as the mean \pm standard error of the mean. Differences were compared with two-tailed Student's t-test using GraphPad Prism software (version 5.0; GraphPad Software, Inc., La Jolla, CA, USA) and P<0.05 was considered to indicate a statistically significant difference.

\section{Result}

Participants. All the patients underwent ultrasonography of the carotid and intracranial arteries. The diabetic patients were divided into two groups, depending on these ultrasound findings: i) individuals free of atherosclerotic stenosis, the diabetic group and ii) individuals with atherosclerotic stenosis, the diabetic-atherosclerosis group (24). HDLs obtained from diabetic groups prior to NXT therapy were designated D-HDL baseline (BL) and D-atherosclerosis (As)-HDL BL. HDL obtained from the two groups following 3 months of NXT therapy were designated D-HDL M3 and D-As-HDL M3. Baseline demographic and clinical characteristics of the diabetic patients and healthy subjects are presented in Table I. No significant difference was observed in age, gender, body mass index, MAP and lipid profile between the diabetic and diabetic-atherosclerosis groups. The NXT treatment was well-tolerated by all subjects in the two groups and there were no significant side-effects observed during therapy. All the patients underwent the anti-diabetic therapy (insulin or oral 
A
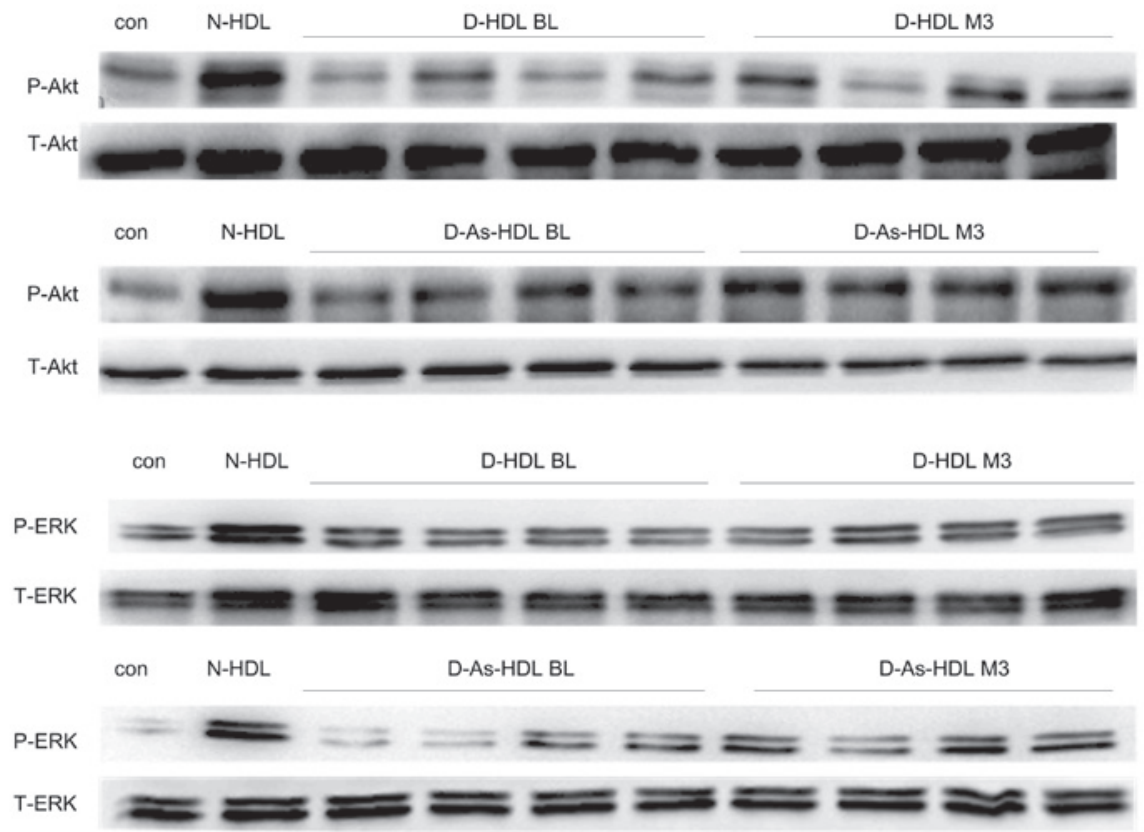

B

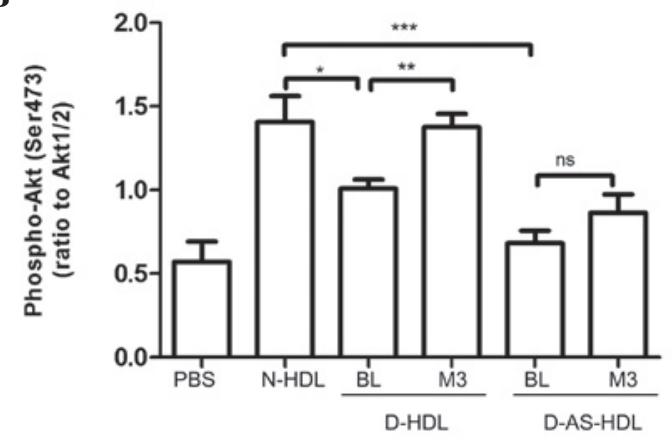

C

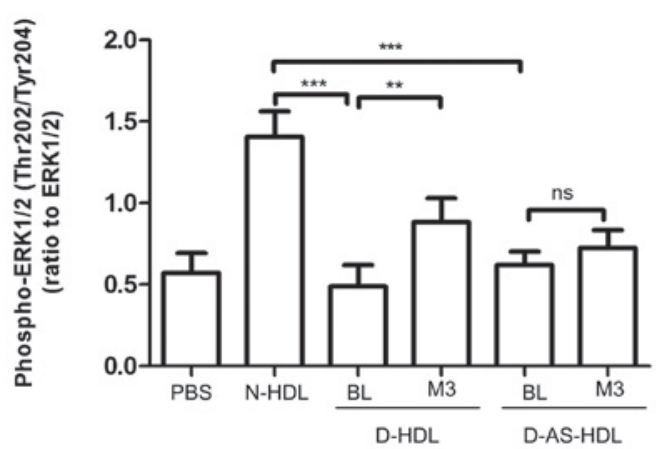

Figure 5. Diminished capacity to activate phosphatidylinositol-4,5-bisphosphate 3-kinase/Akt and MAPK/ERK signaling pathways of D-HDL was reversed by NXT therapy. (A) HUVECs were treated with N-HDL, D-HDL BL, D-HDL M3, D-As-HDL BL, D-As-HDL M3 in 100 mg/ml apolipoprotein A-I for $10 \mathrm{~min}$. Cell lysates were analyzed by western blotting using anti-p-Akt (Ser473) antibody, anti-p-ERK1/2 (Thr202/Tyr204) antibody, anti-total-Akt antibody, and anti-total-ERK1/2 antibody. Density of the (B) p-Akt and (C) p-ERK1/2 bands was normalized to the total-Akt and total-ERK1/2 bands, respectively. ${ }^{*} \mathrm{P}<0.05,{ }^{* *} \mathrm{P}<0.01,{ }^{* * *} \mathrm{P}<0.001$ (Student's t-test). HDL, high-density lipoprotein; NXT, naoxintong; HUVECs, human umbilical vein endothelial cells; N, normal; D, diabetic; BL, baseline; M3, 3 months of NXT; PBS, phosphate-buffered saline; As, atherosclerosis; ns, no significance; p, phosphorylated; ERK, extracellular signal-regulated kinase.

drugs, including metformin, sulfonylurea, $\alpha$-glucosidase and thiazolidinediones). Statins were prescribed to 5 patients of the diabetic-atherosclerosis group and 4 of the diabetic group. Anti-hypertensive therapy was prescribed to 15 patients of the diabetic-atherosclerosis group and to 14 of the diabetic group. Characteristics of the patients in the two groups prior to and following treatment are presented in Table II. Compared with the baseline characteristics, there were no significant changes observed in the lipid profile except for the low density lipoprotein (LDL)-c level in the diabetic-atherosclerosis group, which was decreased $(\mathrm{P}=0.043)$. In addition, the hemoglobin A1c level in patients of the two groups decreased significantly $(\mathrm{P}<0.01)$.

NXT intervention improves proliferative effects of HDL on ECs in diabetic patients. The HUVECs were stimulated by different types of HDL in a time-dependent manner and similar patterns of action were observed in cell proliferation. Normal (N)-HDL (100 $\mu \mathrm{g} / \mathrm{ml}$ apo-AI) demonstrated an increase in relative cell number for $36 \mathrm{~h}$, while D-HDL BL and D-As-HDL BL demonstrated diminished increases. HUVEC proliferation was increased by $27 \%$ in the D-HDL M3 group when compared with the D-HDL BL group at $48 \mathrm{~h}$ (Fig. 1). While the D-As-HDL M3 group demonstrated an increased ability (by 10\%) to stimulate cell proliferation compared with D-As-HDL BL at $48 \mathrm{~h}$ (Fig. 1).

NXT intervention improves D-HDL-associated HUVEC migration. Wound healing and Transwell assays were performed to analyze HUVEC migration (Fig. 2). Compared with N-HDL, D-HDL BL and D-As-HDL BL were less effective (38 and $50 \%$, respectively) at stimulating HUVEC migration in the wound healing assay (Fig. 2A and C). D-HDL M3 stimulated $22 \%$ greater cell migration compared with D-HDL BL, and the D-As-HDL M3 group demonstrated 15\% more migration when compared with D-As-HDL BL group (Fig. 2C).

Results of the Transwell assay were similar. D-HDL M3 had a greater ability (by 35\%) to stimulate cell migration compared 
with D-HDL BL (Fig. 2B and D). The D-As-HDL BL group stimulated less cell proliferation than the D-As-HDL M3 group (Fig. 2D).

NXT therapy improves the endothelial-associated angiogenesis effects of HDL. Compared with N-HDL, D-HDL BL and D-As-HDL BL were 51 and $61 \%$, respectively, less effective in tube formation (Fig. 3A and B). Following 3 months of NXT therapy, the D-HDL M3 and D-As-HDL M3 groups demonstrated increased ability to stimulate cell tube formation (increase of 54 and 30\%, respectively) when compared with the D-HDL BL and D-As-HDL BL groups (Fig. 3B).

NXT therapy improves the anti-apoptotic effects of $D-H D L$. HUVECs exhibited marked apoptosis following TNF- $\alpha$ treatment. N-HDL treatment reduced rates of apoptosis by 45\% (Fig. 4A and B). D-HDL BL and D-As-HDL BL demonstrated reduced ability to protect HUVECs from apoptosis; D-HDL BL and D-As-HDL BL were 48 and 35\%, respectively, less effective when compared with N-HDL (Fig. 4B). Following 3 months of NXT therapy, the D-HDL M3 group exhibited an increased ability (by $34 \%$ ) to inhibit cell apoptosis when compared with D-HDL BL (Fig. 4B). While, the increasing ability in inhibiting cell apoptosis of D-As-HDL M3 was 23\% (Fig. 4B).

HUVECs treated with N-HDL + TNF- $\alpha$ exhibited $48 \%$ less caspase- 3 activity in cell lysates than cells treated with TNF- $\alpha$ alone (Fig. 4C). The D-HDL BL and D-As-HDL BL pretreatment groups exhibited 72 and $64 \%$, respectively more caspase-3 activity when compared with N-HDL (Fig. 4C). Following the 3-month NXT therapy, the D-HDL M3 group exhibited 53\% less caspase-3 activity when compared with D-HDL BL, while no change in the caspase-3 activity level was exhibited in the D-As-HDL M3 group (Fig. 4C).

Diminished capacity of D-HDL to activate Akt and ERK1/2 phosphorylation was abrogated by NXT therapy. Akt and ERK1/2 phosphorylation had been demonstrated to be important in signal transduction for EC proliferation, migration and angiogenesis $(25,26)$. The present study analyzed whetherNXT therapy increased D-HDL function by increasing Akt and ERK1/2 phosphorylation (Fig. 5). Compared with N-HDL, D-HDL BL and D-As-HDL BL demonstrated reduced phosphorylation of Akt (D-HDL BL, 28\%; D-As-HDL BL, 51\%). HUVECs stimulated by D-HDL M3 exhibited 36\% more Akt phosphorylation than those stimulated by D-HDL BL (Fig. 5A and C). HUVECs stimulated by D-HDL M3 exhibited 26\% more Akt phosphorylation than those stimulated by D-HDL BL. However, no significant difference in Akt phosphorylation was observed between HUVECs stimulated by D-As-HDL M3 and those stimulated by D-As-HDL BL (Fig. 5A and C).

ERK1/2 phosphorylation was also observed. Similarly, compared with N-HDL, D-HDL BL and D-As-HDL BL reduced phosphorylation of ERK1/2 by 34 and 26\%, respectively (Fig. 5B and D). HUVECs stimulated by D-HDL M3 demonstrated $80 \%$ greater ERK $1 / 2$ phosphorylation than those stimulated by D-HDL BL (Fig. 5B and D). HUVECs stimulated with D-As-HDL M3 exhibited 16\% more ERK1/2 phosphorylation when compared with those stimulated by D-As-HDL BL (Fig. 5B and D).

\section{Discussion}

The present study indicated that HDL from patients with T2DM exhibited a reduced capacity to stimulate EC proliferation, migration, and anti-apoptotic and angiogenesis functions, which are markedly different from the effects observed with HDL from healthy subjects. Furthermore, HDL from patients with T2DM at early and late stages of the disease present a progressive loss of ability. Notably, NXT therapy improved the protective properties of HDL isolated from diabetic patients on endothelial function as, following NXT therapy, HDL from diabetic patients exhibited an improved capacity to stimulate EC proliferation and migration. In addition, HUVECs treated with D-HDL M3 and D-As-HDL M3 demonstrated increased Akt and ERK phosphorylation, which may explain the increase in migration. Therefore, NXT therapy may be a promising strategy for restoring the direct protective functions of HDL on ECs, rather than increasing HDL plasma levels.

HDL protects against atherosclerosis by reverse cholesterol transportation, anti-inflammatory and anti-oxidative effects, and endothelial protection. However, it has previously been observed that the effects of HDL are heterogeneous (27). Modifying HDL with myeloperoxidase improves the proliferative and migratory functions of ECs (28). Furthermore, HDL from coronary heart disease patients exert a pro-inflammatory effect rather than an anti-inflammatory effect (27). HDL particles undergo modifications during the development of diabetes and the function of D-HDL is controversial (11). D-HDL loses the ability to inhibit oxidized LDL-induced vascular damage (29). The abnormalities of D-HDL are triglyceride enrichment $(30,31)$ and glycation of apoA-I (32). However, regarding the benefits of the phospholipid components of HDL, HDL obtained from early-stage diabetes patients exerts enhanced effect in activating the cyclooxygenase-2/prostacyclin signaling pathway compared with healthy controls (33). In the present study, HDL from T2DM patients lost the capacity to stimulate EC proliferation and migration, which was consistent with a previous study (10). Furthermore, the anti-apoptotic and angiogenesis ability of D-HDL was investigated, which, to the best of our knowledge, has not yet been analyzed. In the Matrigel angiogenesis assay, D-HDL demonstrated 51 to $61 \%$ reduced tube length when compared with N-HDL. The HDL receptor, SR-BI is required for the action on EC. The downstream process involves proto-oncogene tyrosine-protein kinase Src activation and results in parallel activation of the Akt kinase and mitogen-activated kinases. A previous study demonstrated that cell surface levels of SR-BI were decreased following incubation with D-HDL when compared with N-HDL (10). LY294002, a phosphatidylinositol-4,5-bisphosphate 3-kinase (PI3K) inhibitor, and PD98059, a MEK inhibitor, inhibit HDL-induced EC migration (28). The present study suggests that NXT therapy restores D-HDL function. Notably, HDL from different diabetes patients demonstrated different responses to NXT therapy. The majority of patients in the diabetes group had the disease for $\leq 5$ years. However, in the diabetes group with atherosclerosis, the majority of patients had the disease for $\geq 10$ years. In the diabetes group with atherosclerosis, no significant change was observed between D-As-HDL BL and D-As-HDL M3 in the migration and tube formation assays. Although the present study did not 
investigate whether these two types of HDL possessed any chemical differences, long-time glycation and an oxidative and pro-inflammatory condition in these atherosclerosis patients may result in modifications to HDL that cannot be reversed by the short-term therapy used in the present study. Future studies on a larger sample size and with a longer intervention period are required to demonstrate whether treatments at different stages of diabetes have the potential to modulate HDL function.

The main components of NXT include Radix Astragali, Angelicae Sinensis, Radix Paeoniae Rubra and Ligusticum wallichii. Radix Astragali exerts immune-regulating effects and a previous study indicated an Astragalus polysaccharide suppresses the expression of adhesion molecules via the regulation of the p38 MAPK signaling pathway in ECs (34). Radix Astragali, the major component of NXT, has also been reported to exhibit antioxidant activities to prevent LDL oxidation (35). Previous studies demonstrate that NXT therapy had lipid lowering effects (36), anti-inflammatory effects $(37)$, and anti-platelet potential $(38,39)$ in atherosclerosis patients. In the present study, a novel therapeutic target of NXT therapy was investigated. Following the 3-month NXT therapy, D-HDL demonstrated an increased ability to stimulate HUVEC proliferation and migration. However, the mechanism by which NXT therapy exerts these effects on the endothelial function of HDL remains to be elucidated.

In conclusion, the present study demonstrates that HDL from diabetic patients has a markedly impaired protective effect on endothelial function in vitro and that the level of impairment is dependent on the stage of diabetes. The impaired function was partly abrogated by NXT therapy.

\section{Acknowledgements}

The present study was supported by a 'Major New Drug Development' grant from the National S\&T Major Project of China (grant. no. 2008ZX09312-017), a '973' National S\&T Major Project grant (grant no. 2011CB503900), the National Natural Science Foundation of China (grant nos. 81370235 and 81170101) and by the Natural Science Foundation of Beijing (grant no. 7122106).

Dr Yining Huang and Dr Lemin Zheng are the guarantors of the present study, and take full responsibility for the integrity of the data and the accuracy of data analysis.

\section{References}

1. Woodman RJ, Chew GT and Watts GF: Mechanisms, significance and treatment of vascular dysfunction in type 2 diabetes mellitus: Focus on lipid-regulating therapy. Drugs 65: 31-74, 2005.

2. Terasaka N, Wang N, Yvan-Charvet L and Tall AR: High-density lipoprotein protects macrophages from oxidized low-density lipoprotein-induced apoptosis by promoting efflux of 7-ketocholesterol via ABCG1. Proc Natl Acad Sci USA 104: 15093-15098, 2007.

3. Tauber JP, Cheng J and Gospodarowicz D: Effect of high and low density lipoproteins on proliferation of cultured bovine vascular endothelial cells. J Clin Invest 66: 696-708, 1980.

4. Tauber JP, Cheng J, Massoglia S and Gospodarowicz D: High density lipoproteins and the growth of vascular endothelial cells in serum-free medium. In Vitro 17: 519-530, 1981.

5. Tamagaki T, Sawada S, Imamura H, Tada Y, Yamasaki S, Toratani A, Sato T, Komatsu S, Akamatsu N, Yamagami M, et al: Effects of high-density lipoproteins on intracellular $\mathrm{pH}$ and proliferation of human vascular endothelial cells. Atherosclerosis 123: 73-82, 1996.
6. Sugano M, Tsuchida K and Makino N: High-density lipoproteins protect endothelial cells from tumor necrosis factor-alpha-induced apoptosis. Biochem Biophys Res Commun 272: 872-876, 2000.

7. Zheng L, Settle M, Brubaker G, Schmitt D, Hazen SL, Smith JD and Kinter M: Localization of nitration and chlorination sites on apolipoprotein A-I catalyzed by myeloperoxidase in human atheroma and associated oxidative impairment in ABCA1-dependent cholesterol efflux from macrophages. J Biol Chem 280: 38-47, 2005.

8. Nobecourt E, Jacqueminet S, Hansel B, Chantepie S, Grimaldi A, Chapman MJ and Kontush A: Defective antioxidative activity of small dense HDL3 particles in type 2 diabetes: Relationship to elevated oxidative stress and hyperglycaemia. Diabetologia 48: $529-538,2005$.

9. Sorrentino SA, Besler C, Rohrer L, Meyer M, Heinrich K, Bahlmann FH, Mueller M, Horváth T, Doerries C, Heinemann M, et al: Endothelial-vasoprotective effects of high-density lipoprotein are impaired in patients with type 2 diabetes mellitus but are improved after extended-release niacin therapy. Circulation 121: 110-122, 2010.

10. Pan B, Ma Y, Ren H, He Y, Wang Y, Lv X, Liu D, Ji L, Yu B, Wang Y, et al: Diabetic HDL is dysfunctional in stimulating endothelial cell migration and proliferation due to down regulation of SR-BI expression. PLoS One 7: e48530, 2012.

11. Lüscher TF, Landmesser U, von Eckardstein $A$ and Fogelman AM: High-density lipoprotein: Vascular protective effects, dysfunction, and potential as therapeutic target. Circ Res 14: 171-182, 2014.

12. Rader DJ: Molecular regulation of HDL metabolism and function: Implications for novel therapies. J Clin Invest 116: 3090-3100, 2006.

13. Yvan-Charvet L, Matsuura F, Wang N, Bamberger MJ, Nguyen T, Rinninger F, Jiang XC, Shear CL and Tall AR: Inhibition of cholesteryl ester transfer protein by torcetrapib modestly increases macrophage cholesterol efflux to HDL. Arterioscler Thromb Vasc Biol 27: 1132-1138, 2007.

14. Chen C, Venketasubramanian N, Gan RN, Lambert C, Picard D, Chan BP, Chan E, Bousser MG and Xuemin S: Danqi Piantang Jiaonang (DJ), a traditional Chinese medicine, in poststroke recovery. Stroke 40: 859-863, 2009.

15. Zhang H, Wang WR, Lin R, Zhang JY, Ji QL, Lin QQ and Yang LN: Buyang Huanwu decoction ameliorates coronary heart disease with Qi deficiency and blood stasis syndrome by reducing CRP and CD40 in rats. J Ethnopharmacol 130: 98-102, 2010.

16. Chen DK, Zhang HQ and Zhang JH: Intervening effect of naoxintong on anti-platelet treatment with aspirin. Zhongguo Zhong Xi Yi Jie He Za Zhi 28: 843-846, 2008 (In Chinese).

17. Grundy SM, Cleeman JI, Daniels SR, Donato KA, Eckel RH, Franklin BA, Gordon DJ, Krauss RM, Savage PJ, Smith SC Jr, et al: Diagnosis and management of the metabolic syndrome: An American heart association/national heart, lung and blood institute scientific statement. Circulation 112: 2735-2752, 2005.

18. Alberti KG,Zimmet P and Shaw J; IDF Epidemiology Task Force Consensus Group: The metabolic syndrome-a new worldwide definition. Lancet 366: 1059-1062, 2005.

19. Huang B, Li G, Guo YF, Wang SS, Liu F, Xu HY and Yang HJ: Study on absorption location of four components from Naoxintong capsule. Zhongguo Zhong Yao Za Zhi 38: 889-893, 2013.

20. Songsong W, Haiyu X, Yan M, Xuguang W, Yang S, Bin H, Shihuan T, Yi Z, Defeng L, Rixin L, et al: Characterization and rapid identification of chemical constituents of NaoXinTong capsules by UHPLC-linear ion trap/Orbitrap mass spectrometry. J Pharm Biomed Anal 111: 104-118, 2015.

21. Chung BH, Wilkinson T, Geer JC and Segrest JP: Preparative and quantitative isolation of plasma lipoproteins: Rapid, single discontinuous density gradient ultracentrifugation in a vertical rotor. J Lipid Res 21: 284-291, 1980.

22. Jaffe EA, Nachman RL, Becker CG and Minick CR: Culture of human endothelial cells derived from umbilical veins. Identification by morphologic and immunologic criteria. J Clin Invest 52: 2745-2756, 1973.

23. Miura S, Fujino M, Matsuo Y, Kawamura A, Tanigawa H, Nishikawa $\mathrm{H}$ and Saku K: High density lipoprotein-induced angiogenesis requires the activation of Ras/MAP kinase in human coronary artery endothelial cells. Arterioscler Thromb Vasc Biol 23: 802-808, 2003.

24. Katakami N, Kaneto H and Shimomura I: Carotid ultrasonography: A potent tool for better clinical practice in diagnosis of atherosclerosis in diabetic patients. J Diabetes Investig 5: 3-13, 2014. 
25. Seetharam D, Mineo C, Gormley AK, Gibson LL, Vongpatanasin W, Chambliss KL, Hahner LD, Cummings ML, Kitchens RL, Marce YL, et al: High-density lipoprotein promotes endothelial cell migration and reendothelialization via scavenger receptor-B type I. Circ Res 98: 63-72, 2006.

26. Zhu W, Saddar S, Seetharam D, Chambliss KL, Longoria C, Silver DL, Yuhanna IS, Shaul PW and Mineo C: The scavenger receptor class B type I adaptor protein PDZK1 maintains endothelial monolayer integrity. Circ Res 102: 480-487, 2008.

27. Ansell BJ, Navab M, Hama S, Kamranpour N, Fonarow G, Hough G, Rahmani S, Mottahedeh R, Dave R, Reddy ST and Fogelman AM: Inflammatory/antiinflammatory properties of high-density lipoprotein distinguish patients from control subjects better than high-density lipoprotein cholesterol levels and are favorably affected by simvastatin treatment. Circulation 108: 2751-2756, 2003.

28. Pan B, Yu B, Ren H, Willard B, Pan L, Zu L, Shen X, Ma Y, Li X, Niu C, et al: High-density lipoprotein nitration and chlorination catalyzed by myeloperoxidase impair its effect of promoting endothelial repair. Free Radic Biol Med 60: 272-281, 2013.

29. Persegol L, Verges B, Foissac M, Gambert P and Duvillard L: Inability of HDL from type 2 diabetic patients to counteract the inhibitory effect of oxidised LDL on endothelium-dependent vasorelaxation. Diabetologia 49: 1380-1386, 2006.

30. Biesbroeck RC, Albers JJ, Wahl PW, Weinberg CR, Bassett ML and Bierman EL: Abnormal composition of high density lipoproteins in non-insulin-dependent diabetics. Diabetes 31: 126-131, 1982.

31. Taskinen MR: Quantitative and qualitative lipoprotein abnormalities in diabetes mellitus. Diabetes 41 (Suppl 2): S12-S17, 1992.

32. Nobécourt E, Zeng J, Davies MJ, Brown BE, Yadav S, Barter PJ and Rye KA: Effects of cross-link breakers, glycation inhibitors and insulin sensitisers on HDL function and the non-enzymatic glycation of apolipoprotein A-I. Diabetologia 51: 1008-1017, 2008 .
33. Tong X, Lv P, Mathew AV, Liu D, Niu C, Wang Y, Ji L, Li J, Fu Z, Pan B, et al: The compensatory enrichment of sphingosine -1-phosphate harbored on glycated high-density lipoprotein restores endothelial protective function in type 2 diabetes mellitus. Cardiovasc Diabetol 13: 82, 2014.

34. Hai-Yan Z, Yong-Hong G, Zhi-Yao W, Bing X, Ai-Ming W, Yan-Wei X, Bei L, Li-Xia and Li-Xin C: Astragalus polysaccharide suppresses the expression of adhesion molecules through the regulation of the p38 MAPK signaling pathway in human cardiac microvascular endothelial cells after ischemia-reperfusion injury. Evid Based Complement Alternat Med 2013: 280493, 2013

35. Chan JY, Koon JC, Leung PC, Che CT and Fung KP: Suppression of low-density lipoprotein oxidation, vascular smooth muscle cell proliferation and migration by a herbal extract of Radix Astragali, Radix Codonopsis and Cortex Lycii. BMC Complement Altern Med 11: 32, 2011.

36. Zhao J, Zhu H, Wang S, Ma X, Liu X, Wang C, Zhao H, Fan S, Jin X, Zhao B, et al: Naoxintong protects against atherosclerosis through lipid-lowering and inhibiting maturation of dendritic cells in LDL receptor knockout mice fed a high-fat diet. Curr Pharm Des 19: 5891-5896, 2013.

37. Shin HY, Shin TY, An NH, Kim HR, Chae HJ, Kim YK, Um JY, Hong SH and Kim HM: The immunosuppressive effect of Buchang-tang through inhibition of mitogen-activated protein kinase and nuclear factor activation in MOLT-4 cells. J Ethnopharmacol 102: 95-101, 2005.

38. Chen H, Yu G, Sun H, Wu X and Wang H: Comparison of adjunctive naoxintong versus clopidogrel in volunteers with the CYP2C19*2 gene mutation accompanied with qi deficiency and blood stasis constitution. Evid Based Complement Alternat Med 2011: 207034, 2011.

39. Chen H, Zhang Y, Wu X, Li C and Wang H: In Vitro assessment of cytochrome P450 2C19 potential of naoxintong. Evid Based Complement Alternat Med 2012: 430262, 2012. 